\title{
First measurements of muon anti-neutrino disappearance by the T2K experiment
}

\author{
Takahiro HIRAKI* for the T2K collaboration \\ Kyoto University \\ E-mail: hiraki@scphys.kyoto-u.ac.jp
}

The T2K experiment is a long-baseline neutrino oscillation experiment. Last year we started to take data with anti-neutrino beam. In this poster proceedings the first result of $\bar{v}_{\mu}$ disappearance analysis is shown. We fit the muon-like events observed at Super-Kamiokande when running in anti-neutrino mode to estimate the oscillation parameters of the PMNS model. We describe the analysis overview and present systematic errors, preliminary confidence intervals and best-fit points obtained with anti-neutrino mode data.

Flavor Physics \& CP Violation 2015,

May 25-29, 2015

Nagoya, Japan

* Speaker. 


\section{Introduction}

The T2K experiment is a long-baseline neutrino oscillation experiment [1]. An intense muon neutrino beam is produced by striking $30 \mathrm{GeV}$ protons on a graphite target, producing secondary hadrons which are focused by magnetic horns. The produced neutrinos are measured by the offaxis near detectors (ND) at J-PARC and by the Super-Kamiokande detector (SK), which is $295 \mathrm{~km}$ away from J-PARC.

Using the data collected until 2013, T2K published measurements of $v_{\mu}$ disappearance and $v_{e}$ appearance with a world-leading precision [2]. In 2014 we started to take data with $\bar{v}$ beam. The $\bar{v}$ beam is obtained by focusing $\pi^{-}$'s with 3 electromagnetic horns.

In this poster proceedings we report the first result of $\bar{v}_{\mu}$ disappearance analysis. One-ring muon-like events at SK are selected. In this analysis, to see potential effects coming from a new physics like the CPT violation or non-standard interactions, we treat $\left(\theta_{23}, \Delta m_{32}^{2}\right)$ for neutrinos and $\left(\bar{\theta}_{23}, \Delta \bar{m}_{32}^{2}\right)$ for anti-neutrinos as independent parameters and fit data with $\left(\bar{\theta}_{23}, \Delta \bar{m}_{32}^{2}\right)$.

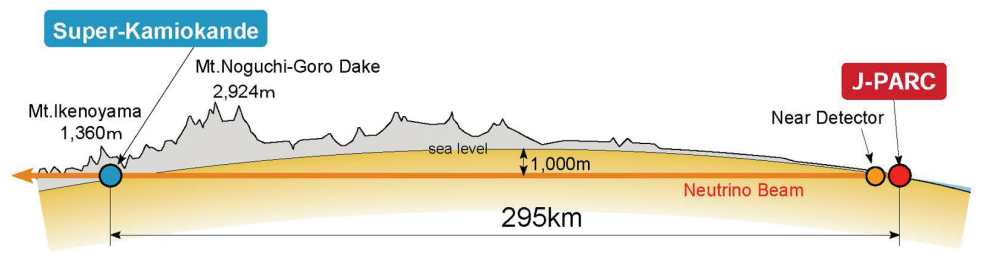

Figure 1: Schematic view of the T2K experiment

\section{Analysis overview}

The analysis is carried out by comparing the observed data to the prediction assuming neutrino oscillation for a given set of values of the neutrino oscillation parameters. The energy spectrum at SK is first predicted with the neutrino flux and the neutrino-nucleus interaction cross section, both of which are modeled based on external data. The ND data including both $v$-mode and $\bar{v}$-mode data is then used to refine the prediction at SK.

As mentioned in the introduction, we treat $\left(\theta_{23}, \Delta m_{32}^{2}\right)$ for neutrinos and $\left(\bar{\theta}_{23}, \Delta \bar{m}_{32}^{2}\right)$ for antineutrinos as independent parameters. The other oscillation parameters are assumed to be the same for neutrinos and anti-neutrinos and these values are fixed and taken from the $v$-mode results from T2K [2] and the Particle Data Group 2014 [3].

In this analysis an extended maximum likelihood method is used. The likelihood is defined as:

$$
\mathscr{L}\left(N_{\text {obs }}, x, o, f\right)=\mathscr{L}_{\text {norm }}\left(N_{\text {obs }}, o, f\right) \times \mathscr{L}_{\text {shape }}\left(N_{\text {obs }}, x, o, f\right) \times \mathscr{L}_{\text {syst }}(f)
$$

where

- $N_{\text {obs }}$ is the number of 1-Ring $\mu$-like candidate $(1 \mathrm{R} \mu)$ events observed in SK

- $x$ represents the reconstructed energy of each event 
- $o$ represents the oscillation parameters we are trying to measure $\left(\bar{\theta}_{23}\right.$ and $\left.\Delta \bar{m}_{32}^{2}\right)$

- $f$ corresponds to the nuisance parameters describing systematic uncertainties.

$\mathscr{L}_{\text {norm }}$ is the normalization term of the likelihood and the probability follows a Poisson distribution. $\mathscr{L}_{\text {shape }}$ is the shape term, and uses the information coming from the shape of the distribution of the reconstructed energy. $\mathscr{L}_{\text {syst }}$ is the systematic term of the likelihood. Systematic parameters used in the analysis are described in Section 3.

Expected $1 \mathrm{R} \mu$ events at SK with $2.3 \times 10^{20}$ protons-on-target (POT) data are listed in Table. 1. Here $\left(\theta_{23}, \Delta m_{32}^{2}\right)=\left(\bar{\theta}_{23}, \Delta \bar{m}_{32}^{2}\right)$ is assumed. Due to the good particle identification capability of the SK detector, $v_{e}$ and $\left(\bar{v}_{e}\right)$ contamination are small. Compared to the $v$-mode case, the fraction of wrong-sign contamination $\left(v_{\mu}\right)$ is larger mainly because anti-neutrino cross section is much smaller than neutrino cross section.

Table 1: expected 1-Ring $\mu$-like events at SK with $2.3 \times 10^{20}$ POT data

\begin{tabular}{ccccc}
\hline \hline & $\bar{v}_{\mu}$ & $v_{\mu}$ & $v_{e}+\bar{v}_{e}$ & total \\
\hline no oscillation & 45.4 & 14.3 & 0.06 & 59.8 \\
maximal disappearance & 12.2 & 7.7 & 0.08 & 19.9 \\
\hline \hline
\end{tabular}

\section{Systematic uncertainties}

As sources of systematic error, the neutrino flux in each flavor, neutrino-nucleus interaction, SK detector, final state interaction of hadrons inside nucleus (FSI) and secondary interaction (SI) are considered.

Table 2 summarizes the uncertainties on number-of-event prediction, where those obtained without or with constraints by the measurement in ND are shown. The uncertainties on the neutrino flux and some cross section parameters are well-constrained by ND data. The dominant systematic uncertainties come from the interaction which could not constraint by the ND measurement because of the difference of the materials between ND (carbon) and SK (oxygen). In near future these errors are expected to be decreased by analyzing $v$-oxygen interaction data in ND.

Table 2: Fractional and total systematic uncertainties with and without ND measurement

\begin{tabular}{|c|c|c|}
\hline \multirow[t]{2}{*}{ Error source } & \multicolumn{2}{|c|}{$1 \sigma$} \\
\hline & without ND measurement & with ND measurement \\
\hline flux & $7.1 \%$ & $3.5 \%$ \\
\hline cross section common to ND & $5.8 \%$ & $1.4 \%$ \\
\hline flux $\times$ cross section common to ND & $9.2 \%$ & $3.4 \%$ \\
\hline cross section (SK only) & \multicolumn{2}{|c|}{$10.0 \%$} \\
\hline SK detector & \multicolumn{2}{|c|}{$3.8 \%$} \\
\hline SK FSI and SI & \multicolumn{2}{|c|}{$2.1 \%$} \\
\hline All & $14.4 \%$ & $11.6 \%$ \\
\hline
\end{tabular}



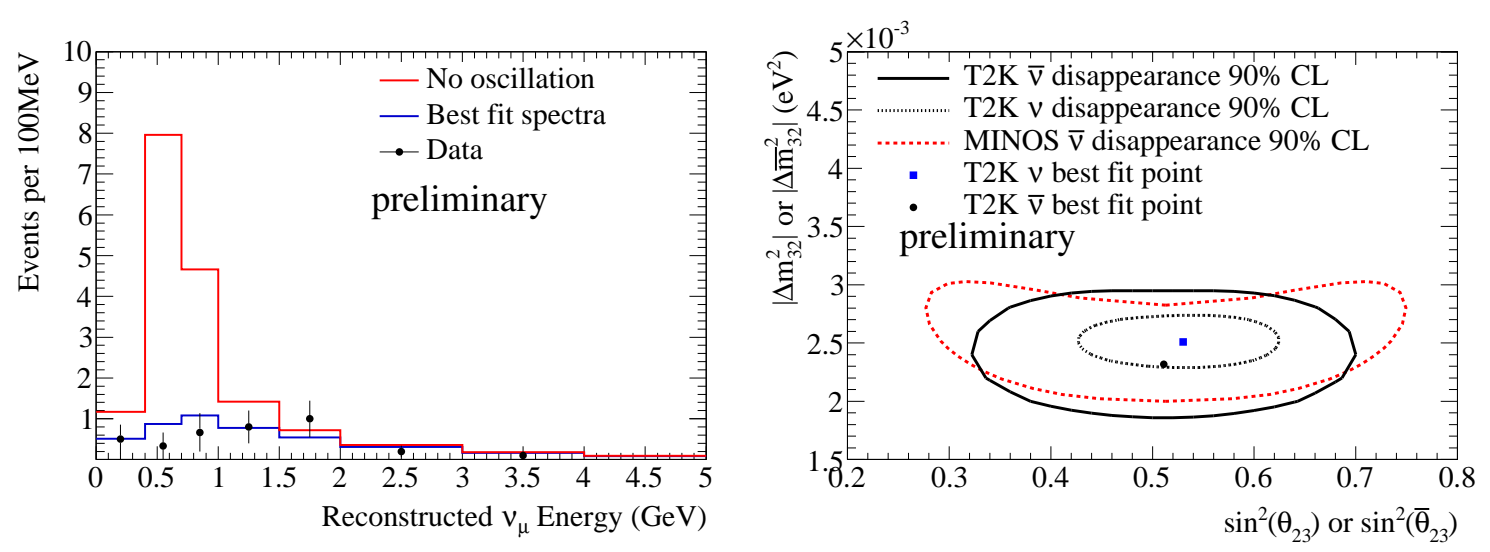

Figure 2: The left plot is the best-fit reconstructed energy spectrum with data points. The right plot is the confidence interval for $v$-mode and $\bar{v}$-mode data.

\section{Oscillation results}

We observed 17 1-Ring $\mu$-like events at SK using $2.3 \times 10^{20}$ POT data. The expected number without oscillation effect is approximately 60 . Thus, clear disappearance is observed.

Figure 2 shows the preliminary result of the fit of $\left(\bar{\theta}_{23}, \Delta \bar{m}_{32}^{2}\right)$ and the best-fit reconstructed energy spectrum in the normal hierarchy.

We obtained the following preliminary best fit and $1 \sigma$ confidence interval:

$\left(\sin ^{2}\left(\bar{\theta}_{23}\right),\left|\Delta \bar{m}_{32}^{2}\right|\right)=\left(0.51 \pm 0.10,2.32 \pm 0.23 \times 10^{-3}\left(\mathrm{eV}^{2}\right)\right)$. The best-fit $\left(\bar{\theta}_{23}, \Delta \bar{m}_{32}^{2}\right)$ is consistent with $\left(\theta_{23}, \Delta m_{32}^{2}\right)$ from the latest T2K $v$-mode measurements. The confidence interval in $\bar{v}$ mode is wider due to lower statistics and larger background.

This result gives a compatible measurement with what was achieved by the MINOS experiment [4].

\section{Conclusion}

The T2K experiment has been taking anti-neutrino mode data since 2014. First measurements of $\bar{v}_{\mu}$ disappearance with a dataset corresponding to $2.3 \times 10^{20}$ POT has been conducted. We obtained the following preliminary best fit and $1 \sigma$ confidence interval: $\left(\sin ^{2}\left(\bar{\theta}_{23}\right),\left|\Delta \bar{m}_{32}^{2}\right|\right)=(0.51 \pm 0.10$, $\left.2.32 \pm 0.23 \times 10^{-3}\left(\mathrm{eV}^{2}\right)\right)$. This result is consistent with the measurement of $\left(\theta_{23}, \Delta m_{32}^{2}\right)$ with $\mathrm{T} 2 \mathrm{~K}$ $v$-mode data [2].

\section{References}

[1] K. Abe et al. (T2K Collaboration): Nucl. Instrum. Methods A659, 106 (2011)

[2] K. Abe et al. (T2K Collaboration): Phys. Rev. D 91, 072010 (2015)

[3] K. A. Olive et al: Chin. Phys. C, 38 (9):090001, (2014)

[4] P. Adamson et al. (MINOS Collaboration): Phys. Rev. Lett, 108, 191801 (2012) 